\title{
The Royal College of Ophthalmologists' National Ophthalmology Database Study of cataract surgery. Report 5: Clinical outcome and risk factors for posterior capsule rupture and visual acuity loss following cataract surgery in patients aged 90 years and older
}

\author{
S. Theodoropoulou ${ }^{1,2} \cdot$ M. T. Grzeda ${ }^{1,3} \cdot$ P. H. J. Donachie ${ }^{4} \cdot$ R. L. Johnston ${ }^{4} \cdot$ J. M. Sparrow ${ }^{1,3} \cdot$ D. M. Tole ${ }^{1}$
}

Received: 3 July 2018 / Revised: 20 December 2018 / Accepted: 25 January 2019 / Published online: 11 March 2019

(c) The Royal College of Ophthalmologists 2019

\begin{abstract}
Background Older age is commonly associated with an increased risk of surgical complications and comparatively poor outcomes.

Purpose To report cataract surgery outcomes and risk indicators for patients aged 90 years and older.

Methods Data collected as part of routine cataract care in 34 centres contributing to the United Kingdom Royal College of Ophthalmologists' National Ophthalmology Database (NOD) were analysed. Very elderly people undergoing cataract surgery were profiled in terms of demographics, pre- and postoperative best-measured visual acuity (VA), ocular co-morbidities, intraoperative posterior capsule rupture (PCR) or vitreous loss or both, and risk indicators for operative PCR and adverse VA outcome.

Results 25,856 cataract operations in 19,166 people of 90 years or older between 2000 and 2014 are reported. Preoperative VA was available for $82.4 \%$ eyes, being $0.30 \mathrm{LogMAR}$ or better in $21.5 \%$. Postoperative VA was available for $61.8 \%$ eyes, being $0.30 \operatorname{LogMAR}$ or better in $74.4 \%$. For those without ocular co-morbidity, postoperative VA was 0.30 LogMAR or better in $84.7 \%$. Various co-morbidities were present in $49 \%$ and contributed to an adverse VA outcome. PCR data were available for all operations and occurred in $2.7 \%$. Significant risk indicators for PCR included pseudoexfoliation/phakodonesis, mature cataract, smaller pupil and worse preoperative VA.

Conclusions Slightly poorer cataract surgery outcome results were noted in patients of 90 years or older, more so in patients with ocular co-morbidity which was highly prevalent. However, surgeons should not be deterred from offering cataract surgery to the very elderly as successful visual rehabilitation remains achievable.
\end{abstract}

Supplementary information The online version of this article (https:// doi.org/10.1038/s41433-019-0389-z) contains supplementary material, which is available to authorized users.

$\triangle$ D. M. Tole

Derek.Tole@uhbristol.nhs.uk

Bristol Eye Hospital, Lower Maudlin Street, Bristol BS1 2LX, UK

2 Department of Ophthalmology, Translational Health Sciences, Bristol Medical School, University Walk BS8 1TD, UK

3 Population Health Sciences, Bristol Medical School, University of Bristol, Canynge Hall, 39 Whatley Road, Bristol BS8 2PS, UK

4 Gloucestershire Hospitals NHS Foundation Trust, Cheltenham General Hospital, Sandford Road, Cheltenham GL53 7AN, UK

\section{Introduction}

The risk of complications associated with many surgical procedures is positively correlated with age, and surgical outcomes are negatively correlated with age [1]. With rapidly ageing populations and greater life expectancy, the number of very elderly patients undergoing cataract surgery will inevitably increase [2].

Although cataract formation is the most significant cause of reversible visual impairment [2], undertaking cataract surgery is dependent upon many factors, including patients' age. Visual impairment in the elderly may impact psychologically and exacerbate pre-existent confusional status e.g. Alzheimer's disease. Even when changes in behaviour are recognised as being due to cataracts, concerns about a possible poor outcome in people of advanced age 
may result in families discouraging surgery [2]. It is of note that very elderly patients may not be being offered the same level of medical and surgical services as their younger counterparts. It has been reported that older patients, who attend the emergency department, are assigned a lower priority level than would otherwise have been required [3]. Moreover, although seniors and surgeons recognise the high value of quality of life at the end of life, this notion is difficult to incorporate into acute surgical decisions, and surgeons may decide that very frail elderly patients should not have surgery [4]. In ophthalmology, pre-existing ocular and general co-morbidity and a short life expectancy may deter some ophthalmologists from offering cataract surgery to very elderly patients, in part because cataract surgery in this population can be technically challenging as many have dense nuclear cataracts [5]. Additional surgical difficulty can occur as a result of elderly patients' not being able to lie flat because of neck or back problems with positioning issues compounded by patient discomfort during a procedure under local anaesthesia [5]. Of note, it has been reported that older adults with dementia are less likely than their peers to undergo cataract surgery [6].

To date, there have been relatively few publications on cataract surgery in patients older than 90 years $[1,2,5,7]$. Reports on outcome in these very elderly people are conflicting as to whether older age, in itself, is a significant predictor of worse visual acuity outcome or not. Norregaard et al. [8] found worse outcome after cataract surgery after controlling for coexisting ocular abnormalities and preoperative visual acuity (VA). However, Lundstrom et al. [7] found that age as a single variable was not associated with a worse outcome when data were adjusted for ocular co-morbidities. Sparrow et al. reported that people aged 90 years or older were less likely than younger individuals to achieve a 'good' VA outcome (LogMAR 0.30 or better), experienced less VA improvement from surgery and were more likely to suffer a significant reduction in VA from preto postoperatively, after adjustment for other risk factors [9]. Narendran et al. also reported that people of 90 years or more were more likely to experience an intraoperative surgical complication than younger patients [10].

The primary aims of the present study was to analyse the clinical outcome following cataract surgery in this age group of patients older than 90 years of age from 34 UK NHS surgical centres, and to determine possible risk factors for operative complications and worse VA outcome after surgery.

\section{Methods}

In this database study, anonymised data on cataract surgery undertaken between January 2000 and December 2014 were obtained from 34 cataract surgery centres as a collaborative exercise with the United Kingdom Royal College of Ophthalmologists' National Ophthalmology Database (NOD). Data on cataract surgery were recorded as part of routine cataract care on the same electronic medical records system across all sites. People aged $\geq 90$ years old who underwent cataract surgery in the 34 surgery sites were included in the analysis. Data in the targeted age group who were undergoing cataract surgery through the 15-year period were analysed. All patient identifiers were removed prior to the data leaving the surgical centres, a 'study ID' being used to link individual people who contributed both eyes to the set of data. The analysis set was therefore anonymous and the study was conducted in accordance with the declaration of Helsinki, the UK's Data Protection Act of 1998 and the General Data Protection Regulation (GDPR) of the European Union.

Data for analysis included patient demographics, first or second eye surgery, preoperative and postoperative VA, presence of ocular co-morbidities and the occurrence of the intraoperative complication of posterior capsule rupture (PCR) or vitreous loss or both (abbreviated as PCR). Preoperative 'best-measured' VA was defined as the better measurement of corrected or uncorrected distance VA. The VA closest to the date of surgery (including the day of surgery and within 90 days prior to surgery) was chosen if more than one VA measurement was available. Pinhole visual acuity measurements were only used for preoperative VA if no other VA measurement was available. Postoperative 'best-measured' VA was defined as the best measurement of corrected distance visual acuity, uncorrected distance visual acuity or pinhole visual acuity (within 14 days and 4 months of surgery). Where VA had been recorded as counting fingers (CF), hand movements (HM), perception of light (PL), and no perception of light (NPL) these were converted for analysis to LogMAR 2.10, 2.40, 2.70, and 3.00 respectively [11]. In view of the uncertainty associated with this approach, vision loss was only deemed to have occurred at this extreme end of the scale where the change from pre- to postoperative VA was greater than a doubling of the visual angle, e.g. CF to PL or HM to NPL (as opposed to a doubling or worse as used for the rest of the scale).

Two adverse clinical outcomes were separately subjected to risk factor analysis. These were PCR and VA Loss, defined as a loss of vision of a doubling or worse of the visual angle from pre- to postoperatively. Candidate risk factors were those recorded preoperatively on the patient's Electronic Medical Record (EMR), ocular co-morbidities being defined as any coexisting ocular or visual pathway disease identified preoperatively that was likely to limit the final VA outcome of surgery, i.e. considered to be a reason for a guarded visual prognosis either directly or as a result of a higher risk of a visually damaging intraoperative adverse event. 
The methods applied for modelling of risk factors for PCR and VA Loss included both bivariate tabular and multivariable logistic regression. Before proceeding to the analyses, all risk factors were grouped into theoretical blocks, each reflecting clinically cohesive themes. Three such blocks were assumed for the current study. (i) Basic patient and eye characteristics: gender, left or right side, first or second eye operation, preoperative VA, systemic alphablocker use; (ii) Ocular co-morbidities and (iii) operative issues: inability to lie flat, pupil size etc. A full list of candidate predictors within each theoretical block is presented in Table 1. Each of these pre-selected candidate variables was subjected to an initial bivariate screening exercise seeking associations by means of Pearson chisquare test. Associations at $p<0.10$ were taken forward for regression with model construction proceeding by sequentially entered candidate risk factors in the groups noted above. Predictors with a $p$-value $<0.05$ were accepted in the multivariable analyses. The regression model employed was multilevel logistic with eyes/operations nested within patients, thus accounting for correlation between eyes within patients [12].

\section{Missing data}

Recording of the presence or absence of a co-morbidity and the occurrence or otherwise of an operative complication are mandated in the EMR system which increases confidence in the completeness of these data items. Absence of recording of clinical features on the EMR was inferred as the absence of a feature. Based on this approach, the only variable with a relatively large proportion of missing data was VA, at both pre- and postoperative time points. Whilst we cannot guarantee that every case was recorded, we would expect that the vast majority were, as the data we have used are the hospital EMR record itself. Where there may have been cases missed out it seems unlikely that older patients would have been selectively affected. Since missing data are known to be a serious problem that potentially can lessen the validity of the statistical analyses (if restricted to complete cases) it was decided to tackle this issue by applying a multiple imputation technique (for details see supplementary online material). Risk models were developed in parallel on datasets including only complete cases and after application of multiple imputation techniques. Results of both approaches are reported in the current paper.

\section{Results}

Data were available for analysis on 25,856 cataract operations performed in 19,166 patients of 90 years of age or older at 34 NHS trusts between January 2000 and December
2014. The baseline characteristics of the study population are included in Table 1 . Women represented $67.0 \%$ of our study population. About $55.5 \%$ of included operations $(n=14,340)$ were for first-eye surgery.

\section{Preoperative features}

The presence or absence of ocular co-morbidity, considered to be a reason for a guarded visual prognosis in the operated eye, was recorded in all cases. At least one reason for a guarded visual prognosis was identified preoperatively in $12,687(49.1 \%)$ eyes. The spectrum and frequency of co-morbidities and surgical risk factors for PCR or VA Loss are shown in Table 1 . The most frequently recorded preoperative candidate indicators of possible surgical risk were a small pupil in $6.5 \%$ eyes and a mature cataract (brunescent/white cataract) in $6.2 \%$ eyes.

A preoperative 'best-measured' VA for the operated eye was available for $21,300(82.4 \%)$ eyes. The mean (SD) preoperative VA was $0.76(0.58) \operatorname{LogMAR}$ and the median was 0.60 LogMAR, including 1285 eyes with CF vision, 757 with HM, 137 with PL and 14 with NPL. Table 2 shows the preoperative best-measured VA for all eyes stratified into VA groups. Preoperative visual acuity was 0.30 LogMAR or better in $21.5 \%$ eyes with available data.

\section{Postoperative features}

A postoperative 'best-measured' VA for the operated eye was available for $15,981(61.8 \%)$ eyes. The postoperative visual acuities in the operated eyes are tabulated in Table 2. Overall, VA improved significantly following cataract surgery with $74.4 \%$ of all eyes with available postoperative data achieving VA of 0.30 LogMAR or better. Relatively few such eyes, $2.3 \%$ achieved VA better than 0.00 LogMAR. Prior to collapse of the VA data for tabulation, of 13,190 eyes with VA at both time points $85 \%$ had better VA postoperatively, with no VA difference in $10 \%$ and with $6 \%$ of eyes having worse VA postoperatively compared with preoperatively. Preoperative VA was similar between eyes with and without a postoperative VA, the preoperative mean (SD) VA for eyes with a postoperative VA was 0.73 (0.55) LogMAR and for eyes without a postoperative VA was $0.80(0.62)$ LogMAR. Table 2 is formatted to provide a cross tabulation of pre- vs. postoperative VA.

Visual outcomes were separately assessed for patients with or without ocular co-morbidities, see Table 3. For eyes without ocular co-morbidity, postoperative VA was 0.30 LogMAR or better in $84.7 \%$ of operated eyes, whereas in eyes with co-morbidity VA 0.30 LogMAR or better was achieved only in $63.6 \%$. 
Table 1 Patient and eye characteristics

\begin{tabular}{|c|c|c|c|c|c|}
\hline & $\begin{array}{l}N(\%) \text { of operations } \\
\text { in group }\end{array}$ & $\begin{array}{l}N(\%) \text { of operations within } \\
\text { group resulting in } \mathrm{PCR}^{\mathrm{a}}\end{array}$ & $p$-value ${ }^{\mathrm{b}}$ & $\begin{array}{l}N(\%) \text { of operations within } \\
\text { group resulting in VA loss }\end{array}$ & $p$-value ${ }^{b}$ \\
\hline \multicolumn{6}{|c|}{ Basic characteristics of operated eyes/patients } \\
\hline Female patients & $17,310(67.0 \%)$ & $473(2.73 \%)$ & 0.258 & $181(2.05 \%)$ & 0.344 \\
\hline Male patients & $8546(33.0 \%)$ & $213(2.49 \%)$ & & $101(2.31 \%)$ & \\
\hline Right eye & $13,082(50.6 \%)$ & $362(2.77 \%)$ & 0.248 & $139(2.06 \%)$ & 0.509 \\
\hline Left eye & $12,774(49.4 \%)$ & $324(2.54 \%)$ & & $143(2.22 \%)$ & \\
\hline First eye & $14,340(55.5 \%)$ & $413(2.88 \%)$ & 0.011 & $177(2.16 \%)$ & 0.801 \\
\hline Second eye & $11,516(44.5 \%)$ & $273(2.37 \%)$ & & $105(2.10 \%)$ & \\
\hline \multicolumn{6}{|l|}{ Best-measured pre-op VA (LogMAR) } \\
\hline $\mathrm{VA}<0.00$ & $24(0.1 \%)$ & $0(0.0 \%)$ & $<0.001$ & $5(20.83 \%)$ & $<0.001$ \\
\hline $\mathrm{VA} \geq 0.00$ to 0.30 & $4551(17.6 \%)$ & $99(2.2 \%)$ & & $68(1.49 \%)$ & \\
\hline $\mathrm{VA}>0.30$ to 0.60 & $8678(33.6 \%)$ & $182(2.1 \%)$ & & $79(0.91 \%)$ & \\
\hline $\mathrm{VA}>0.60$ to 0.90 & $3408(13.2 \%)$ & $87(2.6 \%)$ & & $48(1.41 \%)$ & \\
\hline $\mathrm{VA}>0.90$ to 1.20 & $1600(6.2 \%)$ & $43(2.7 \%)$ & & $33(2.06 \%)$ & \\
\hline $\mathrm{VA}>1.20$ & $3039(11.8 \%)$ & $155(5.1 \%)$ & & $49(1.61 \%)$ & \\
\hline Missing data on VA & $4556(17.6 \%)$ & $120(2.6 \%)$ & & - & \\
\hline Alpha-blocker use & $1496(5.8 \%)$ & $47(3.14 \%)$ & 0.226 & $21(2.60 \%)$ & 0.350 \\
\hline No alpha-blocker use & $24,360(94.2 \%)$ & $639(2.62 \%)$ & & $261(2.11 \%)$ & \\
\hline \multicolumn{6}{|l|}{ Ocular co-morbidities } \\
\hline Age-related macular degeneration & $7234(28.0 \%)$ & $186(2.57 \%)$ & 0.609 & $119(3.03 \%)$ & $<0.001$ \\
\hline $\begin{array}{l}\text { No age-related macular } \\
\text { degeneration }\end{array}$ & $18,622(72.0 \%)$ & $500(2.68 \%)$ & & $163(1.76 \%)$ & \\
\hline Glaucoma & $2960(11.4 \%)$ & $85(2.87 \%)$ & 0.432 & $46(3.30 \%)$ & 0.002 \\
\hline No glaucoma & $22,896(88.6 \%)$ & $601(2.62 \%)$ & & $236(2.00 \%)$ & \\
\hline Corneal pathology & $859(3.3 \%)$ & $32(3.73 \%)$ & 0.047 & $10(2.45 \%)$ & 0.657 \\
\hline No corneal pathology & $24,997(96.7 \%)$ & $654(2.62 \%)$ & & $272(2.13 \%)$ & \\
\hline Uveitis & $147(0.6 \%)$ & $8(5.44 \%)$ & 0.035 & $2(3.64 \%)$ & - \\
\hline No uveitis & $25,709(99.4 \%)$ & $678(2.64 \%)$ & & $280(2.13 \%)$ & \\
\hline Diabetic retinopathy & $512(2.0 \%)$ & $14(2.73 \%)$ & 0.908 & $5(1.91 \%)$ & - \\
\hline No diabetic retinopathy & $25,344(98.0 \%)$ & $672(2.65 \%)$ & & $277(2.14 \%)$ & \\
\hline High myopia & $25,582(1.1 \%)$ & $13(4.74 \%)$ & 0.030 & $0(0.00 \%)$ & - \\
\hline No high myopia & $274(98.9 \%)$ & $673(2.63 \%)$ & & $282(2.16 \%)$ & \\
\hline Amblyopia & $249(1.0 \%)$ & $10(4.02 \%)$ & 0.179 & $1(0.80 \%)$ & - \\
\hline No amblyopia & $25,607(99.0 \%)$ & $676(2.64 \%)$ & & $281(2.15 \%)$ & \\
\hline $\begin{array}{l}\text { Optic nerve or central nervous } \\
\text { system disease }\end{array}$ & $91(0.3 \%)$ & $2(2.20 \%)$ & - & $3(6.38 \%)$ & - \\
\hline $\begin{array}{l}\text { No optic nerve or central nervous } \\
\text { system disease }\end{array}$ & $25,765(99.7 \%)$ & $684(2.65 \%)$ & & $279(2.12 \%)$ & \\
\hline Inherited eye disease & $6(<0.1 \%)$ & $0(0.0 \%)$ & - & $0(0.00 \%)$ & - \\
\hline No inherited eye disease & $25,850(100.0 \%)$ & $686(2.65 \%)$ & & $282(2.14 \%)$ & \\
\hline Other macular pathology & $197(0.8 \%)$ & $5(2.54 \%)$ & 0.920 & $2(1.79 \%)$ & - \\
\hline No other macular pathology & $25,659(99.2 \%)$ & $681(2.65 \%)$ & & $280(2.14 \%)$ & \\
\hline Other retinal pathology & $313(1.2 \%)$ & $10(3.19 \%)$ & 0.548 & $5(3.23 \%)$ & 0.346 \\
\hline No other retinal pathology & $25,543(98.8 \%)$ & $676(2.65 \%)$ & & $277(2.13 \%)$ & \\
\hline Previous vitrectomy surgery & $43(0.2 \%)$ & $0(0.0 \%)$ & - & $0(0.00 \%)$ & - \\
\hline No previous vitrectomy surgery & $25,813(99.8 \%)$ & $686(2.66 \%)$ & & $282(2.14 \%)$ & \\
\hline Previous trabeculectomy surgery & $87(0.3 \%)$ & $6(6.90 \%)$ & 0.014 & $0(0.00 \%)$ & - \\
\hline & $25,769(99.7 \%)$ & $680(2.64 \%)$ & & $282(2.14 \%)$ & \\
\hline
\end{tabular}


Table 1 (continued)

\begin{tabular}{|c|c|c|c|c|c|}
\hline & $\begin{array}{l}N(\%) \text { of operations } \\
\text { in group }\end{array}$ & $\begin{array}{l}N(\%) \text { of operations within } \\
\text { group resulting in } \mathrm{PCR}^{\mathrm{a}}\end{array}$ & $p$-value ${ }^{\mathrm{b}}$ & $\begin{array}{l}N(\%) \text { of operations within } \\
\text { group resulting in VA loss }\end{array}$ & $p$-value ${ }^{\mathrm{b}}$ \\
\hline \multicolumn{6}{|l|}{$\begin{array}{l}\text { No previous trabeculectomy } \\
\text { surgery }\end{array}$} \\
\hline Any other ocular co-morbidity & $869(3.4 \%)$ & $36(4.14 \%)$ & 0.005 & $15(3.45 \%)$ & 0.055 \\
\hline No other ocular co- morbidity & $24,987(96.6 \%)$ & $650(2.60 \%)$ & & $267(2.09 \%)$ & \\
\hline Mature cataract & $1604(6.2 \%)$ & $96(5.99 \%)$ & $<0.001$ & $29(3.79 \%)$ & 0.001 \\
\hline No mature cataract & $24,252(93.8 \%)$ & $590(2.43 \%)$ & & $253(2.04 \%)$ & \\
\hline $\begin{array}{l}\text { At least one of above ocular } \\
\text { problems }\end{array}$ & $12,687(49.1 \%)$ & $373(2.94 \%)$ & 0.005 & $185(2.85 \%)$ & $<0.001$ \\
\hline None of above ocular problems & $13,169(50.9 \%)$ & $313(2.38 \%)$ & & $97(1.45 \%)$ & \\
\hline \multicolumn{6}{|l|}{ Other risk factors (operative issues) } \\
\hline Small pupil & $1689(6.5 \%)$ & $78(4.62 \%)$ & $<0.001$ & $20(2.47 \%)$ & 0.344 \\
\hline Medium & $4085(15.8 \%)$ & $126(3.08 \%)$ & & $53(2.49 \%)$ & \\
\hline Large pupil & $20,082(77.7 \%)$ & $482(2.40 \%)$ & & $209(2.04 \%)$ & \\
\hline Unable to lie flat & $226(0.9 \%)$ & $8(3.54 \%)$ & 0.405 & $3(2.26 \%)$ & - \\
\hline Able to lie flat & $25,630(99.1 \%)$ & $678(2.65 \%)$ & & $279(2.14 \%)$ & \\
\hline Pseudoexfoliation/phakodonesis & $513(2.0 \%)$ & $50(9.75 \%)$ & $<0.001$ & $7(2.92 \%)$ & \\
\hline No pseudoexfoliation/phakodonesis & $25,343(98.0 \%)$ & $636(2.51 \%)$ & & $275(2.12 \%)$ & 0.400 \\
\hline
\end{tabular}

${ }^{a}$ Bivariate tabular analyses looking at the association between PCR and each single variable (rows). Analyses on 25,856 eyes of 19,166 patients (the only exception being association between pre-op VA and PCR where cases with missing pre-op VA were excluded leaving 21,300 eyes for analysis)

${ }^{\mathrm{b}} p$-values associated with Pearson chi-square test (tables with cells with $<5$ eyes not analysed)

${ }^{c}$ Bivariate tabular analyses looking at the association between VA Loss and each single variable (rows). Analysis on 13,190 eyes of 10,930 patients

Table 2 Pre- and postoperative LogMAR visual acuity. Complete case analysis (above) and analysis of data with imputed missing values (below) Complete case analysis

\begin{tabular}{|c|c|c|c|c|c|c|c|c|}
\hline \multirow{2}{*}{$\begin{array}{l}N(\%) \\
\text { Preoperative } \\
\text { LogMAR VA: }\end{array}$} & \multicolumn{8}{|c|}{ Postoperative LogMAR VA } \\
\hline & $\mathrm{VA}<0.00$ & $\begin{array}{l}\mathrm{VA} \geq 0.00 \\
\text { to } 0.30\end{array}$ & $\begin{array}{l}\mathrm{VA}>0.30 \\
\text { to } 0.60\end{array}$ & $\begin{array}{l}\mathrm{VA}>0.60 \\
\text { to } 0.90\end{array}$ & $\begin{array}{l}\mathrm{VA}>0.90 \\
\text { to } 1.20\end{array}$ & $\mathrm{VA}>1.20$ & $\begin{array}{l}\text { Missing data } \\
\text { on VA }\end{array}$ & Total \\
\hline $\mathrm{VA}<0.00$ & $3(12.5 \%)$ & $7(29.2 \%)$ & $2(8.3 \%)$ & $0(0.0 \%)$ & $0(0.0 \%)$ & $0(0.0 \%)$ & $12(50.0 \%)$ & $24(100 \%)$ \\
\hline $\mathrm{VA} \geq 0.00$ to 0.30 & $124(2.7 \%)$ & $2620(57.6 \%)$ & $156(3.4 \%)$ & $13(0.3 \%)$ & $6(0.1 \%)$ & $6(0.1 \%)$ & $1626(35.7 \%)$ & $4551(100 \%)$ \\
\hline $\mathrm{VA}>0.30$ to 0.60 & $119(1.4 \%)$ & $4372(50.4 \%)$ & $835(9.6 \%)$ & $98(1.1 \%)$ & $30(0.4 \%)$ & $29(0.3 \%)$ & $3195(36.8 \%)$ & $8678(100 \%)$ \\
\hline $\mathrm{VA}>0.60$ to 0.90 & $29(0.9 \%)$ & $1330(39.0 \%)$ & $449(13.2 \%)$ & $177(5.2 \%)$ & $38(1.1 \%)$ & $44(1.3 \%)$ & $1341(39.4 \%)$ & $3408(100 \%)$ \\
\hline $\mathrm{VA}>0.90$ to 1.20 & $16(1.0 \%)$ & $496(31.0 \%)$ & $193(12.1 \%)$ & $137(8.6 \%)$ & $104(6.5 \%)$ & $56(3.5 \%)$ & $598(37.4 \%)$ & $1600(100 \%)$ \\
\hline $\mathrm{VA}>1.20$ & $11(0.4 \%)$ & $641(21.1 \%)$ & $259(8.5 \%)$ & $152(5.0 \%)$ & $175(5.8 \%)$ & $463(15.2 \%)$ & $1338(44.0 \%)$ & $3039(100 \%)$ \\
\hline Missing data on VA & $64(1.4 \%)$ & $2061(45.2 \%)$ & $389(8.5 \%)$ & $114(2.5 \%)$ & $54(1.2 \%)$ & $109(2.4 \%)$ & $1765(38.7 \%)$ & $4556(100 \%)$ \\
\hline Total & $366(1.4 \%)$ & $11,527(44.6 \%)$ & $2283(8.8 \%)$ & $691(2.7 \%)$ & $407(1.6 \%)$ & $707(2.7 \%)$ & $9875(38.2 \%)$ & $25,856(100 \%)$ \\
\hline \multicolumn{9}{|c|}{ Analysis of data with imputed missing values (number of datasets, $m=50$ each with $N=25,856$ ) } \\
\hline \multicolumn{9}{|l|}{$\%$} \\
\hline $\mathrm{VA}<0.00$ & $63.2 \%$ & $20.0 \%$ & $16.8 \%$ & $0.0 \%$ & $0.0 \%$ & $0.0 \%$ & - & $100 \%$ \\
\hline $\mathrm{VA} \geq 0.00$ to 0.30 & $24.0 \%$ & $56.7 \%$ & $16.3 \%$ & $2.3 \%$ & $0.4 \%$ & $0.2 \%$ & - & $100 \%$ \\
\hline $\mathrm{VA}>0.30$ to 0.60 & $13.8 \%$ & $51.0 \%$ & $27.8 \%$ & $5.7 \%$ & $1.1 \%$ & $0.6 \%$ & - & $100 \%$ \\
\hline $\mathrm{VA}>0.60$ to 0.90 & $7.8 \%$ & $42.3 \%$ & $31.8 \%$ & $13.5 \%$ & $2.6 \%$ & $2.1 \%$ & - & $100 \%$ \\
\hline $\mathrm{VA}>0.90$ to 1.20 & $6.1 \%$ & $32.9 \%$ & $28.8 \%$ & $18.7 \%$ & $8.6 \%$ & $4.8 \%$ & - & $100 \%$ \\
\hline $\mathrm{VA}>1.20$ & $3.8 \%$ & $19.8 \%$ & $26.7 \%$ & $17.9 \%$ & $11.3 \%$ & $20.4 \%$ & - & $100 \%$ \\
\hline Total & $13.2 \%$ & $45.0 \%$ & $25.9 \%$ & $8.9 \%$ & $3.1 \%$ & $3.8 \%$ & - & $100 \%$ \\
\hline
\end{tabular}




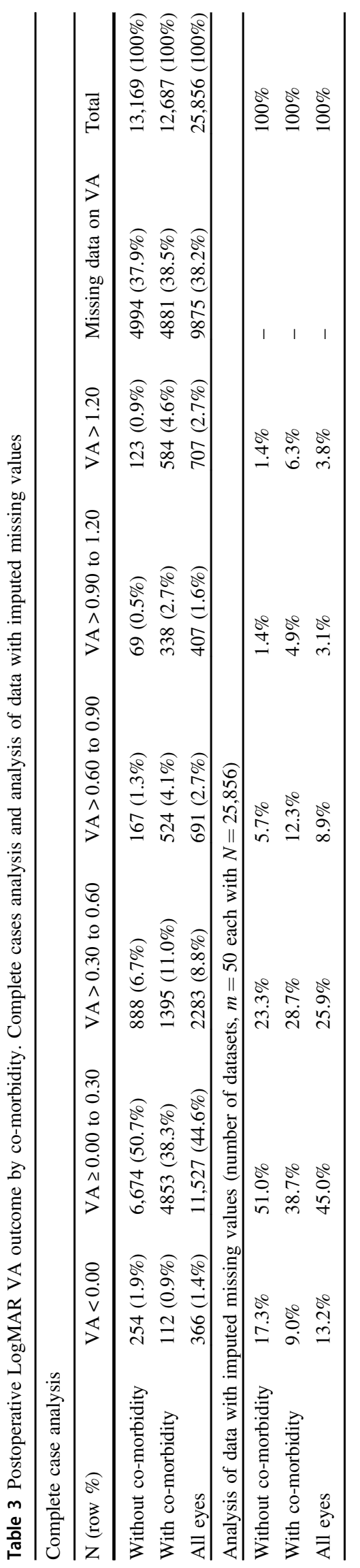

In addition, both missing pre- and postoperative VA data were imputed (see supplementary online information for details) with resultant distributions shown in the lower parts of Tables 2 and 3.

\section{Adverse outcomes}

PCR or vitreous loss or both occurred in 686 (2.7\%) eyes. An initial univariable analysis for potential risk factors was performed (Table 1). Associations in particular for PCR at the level of $p<0.1$ were found for: first-second eye operated, poor preoperative visual acuity, corneal pathology, uveitis, previous trabeculectomy surgery, mature cataract (brunescent/white cataract), high myopia, presence of pseudoexfoliation/phakodonesis, smaller pupil and presence of any other ocular co-morbidity. In the subsequent multivariable logistic regression analysis the presence of pseudoexfoliation/phakodonesis, mature cataract (brunescent/white cataract), a smaller pupil, and poor preoperative VA all significantly increased the risk of PCR in this aged population. Table 4 shows the multivariable logistic regression-derived odds ratios and $95 \%$ confidence intervals for PCR and includes models without and with imputation of missing preoperative VA. Models for PCR developed on complete cases and after multiple imputation of VA data converged to the same set of risk factors.

A similar approach was adopted for candidate risk factors for VA Loss (a doubling or worse of the visual angle following cataract surgery. Table 1 shows the candidate risk factors tested with univariable $p$-values. Associations with VA Loss at the level of $p<0.1$ were found for: preoperative VA, age-related macular degeneration, glaucoma, 'other' ocular co-morbidity and mature cataract (brunescent/white cataract). In the subsequent multivariable logistic regression analysis eyes with age-related macular degeneration, glaucoma and better preoperative VA (LogMAR $\leq 0.3$ i.e. $6 / 12$ or better) were more likely to experience VA Loss, the preoperative VA contrast only being important between certain VA categories. Table 5 shows results of the multivariable logistic regression-derived odds ratios and $95 \%$ confidence intervals for VA Loss following cataract surgery. Models for VA Loss developed for datasets excluding cases with missing values and with missing values imputed converged to the same set of risk factors.

\section{Discussion}

Cataract is a prevalent age-related eye disease and is a leading cause of visual loss in the developing and developed world, particularly in the elderly [13-16]. With greater life expectancy and increasing expectations of good quality 
Table 4 Logistic regressionderived odds ratios (OR) and 95\% confidence intervals $(\mathrm{CI})$ for final model exploring risk factors for Posterior Capsule Rupture (PCR) during cataract surgery

Table 5 Logistic regressionderived odds ratios (OR) and 95\% confidence intervals $(\mathrm{CI})$ for significant risk factors for vision loss (doubling or worse of visual angle) after cataract surgery

\begin{tabular}{|c|c|c|c|c|}
\hline \multicolumn{5}{|c|}{ Risk factors for PCR (Posterior capsule rupture or vitreous loss or both) } \\
\hline & \multicolumn{2}{|c|}{ Complete cases analysis } & \multicolumn{2}{|c|}{$\begin{array}{l}\text { Analysis with imputated } \\
\text { missing values }\end{array}$} \\
\hline & Odds-ratio & $95 \% \mathrm{CI}$ & Odds-ratio & $95 \% \mathrm{CI}$ \\
\hline Presence of pseudoexfoliation/phakodonesis & 3.28 & [2.22 to 4.85$]$ & 3.28 & [2.35 to 4.57$]$ \\
\hline Mature cataract & 1.66 & [1.26 to 2.19$]$ & 1.74 & [1.36 to 2.22$]$ \\
\hline \multicolumn{5}{|l|}{ Pupil size (large pupil as ref) } \\
\hline Medium pupil & 1.32 & [1.06 to 1.66$]$ & 1.26 & [1.03 to 1.53$]$ \\
\hline Small pupil & 1.69 & [1.26 to 2.25$]$ & 1.63 & [1.26 to 2.11$]$ \\
\hline \multicolumn{5}{|l|}{ Visual acuity (LogMAR VA $\leq 0.3$ as ref) } \\
\hline $\mathrm{VA}>0.30$ to 0.60 & 0.96 & [0.75 to 1.24$]$ & 1.22 & [0.82 to 1.81$]$ \\
\hline $\mathrm{VA}>0.60$ to 0.90 & 1.15 & [0.85 to 1.55$]$ & 1.47 & [0.98 to 2.20$]$ \\
\hline $\mathrm{VA}>0.90$ to 1.20 & 1.20 & [0.83 to 1.74$]$ & 1.63 & [1.03 to 2.57$]$ \\
\hline $\mathrm{VA}>1.20$ & 2.19 & [1.66 to 2.88$]$ & 2.75 & [1.81 to 4.18$]$ \\
\hline
\end{tabular}

Complete cases analysis (conducted on dataset excluding cases with missing values; $n=21,300$ eyes/ operations) and analysis of data with imputed missing values ( $m=50$ each with $n=25,856$ eyes/operations)

\begin{tabular}{|c|c|c|c|c|}
\hline \multicolumn{5}{|c|}{ Risk factors for VA Loss (Doubling or worse of visual angle) } \\
\hline & \multicolumn{2}{|c|}{ Complete cases analysis } & \multicolumn{2}{|c|}{$\begin{array}{l}\text { Analysis with imputed missing } \\
\text { values }\end{array}$} \\
\hline & Odds-ratio & $95 \% \mathrm{CI}$ & Odds-ratio & $95 \% \mathrm{CI}$ \\
\hline Age-related macular degeneration & 1.92 & [1.39 to 2.64$]$ & 2.34 & [1.87 to 2.92$]$ \\
\hline Glaucoma & 1.91 & [1.27 to 2.89$]$ & 1.57 & [1.17 to 2.11$]$ \\
\hline \multicolumn{5}{|c|}{ Visual acuity ( $\log$ MAR VA $\leq 0.3$ as ref) } \\
\hline $\mathrm{VA}>0.30$ to 0.60 & 0.49 & {$[0.33$ to 0.74$]$} & 0.60 & [0.43 to 0.84$]$ \\
\hline $\mathrm{VA}>0.60$ to 0.90 & 0.79 & {$[0.50$ to 1.24$]$} & 0.50 & [0.36 to 0.71$]$ \\
\hline $\mathrm{VA}>0.90$ to 1.20 & 1.18 & {$[0.71$ to 1.97$]$} & 0.56 & [0.36 to 0.89$]$ \\
\hline $\mathrm{VA}>1.20$ & 0.93 & [0.59 to 1.48$]$ & 0.31 & [0.20 to 0.47$]$ \\
\hline
\end{tabular}

Complete cases analysis (conducted on dataset excluding cases with missing values; $n=13,190$ eyes/ operations) analysis of data with imputed missing values ( $m=50$ each with $n=25,856$ eyes/operations) of life at all ages, the number of very elderly patients undergoing cataract surgery is expected to grow substantially [17]. To date there has been a lack of data on the outcome of cataract surgery in this age group. Reported data to date include the study of Guzek et al., which had only 84 patients $>80$ years of age in a group of 1000 cases of cataract surgery [18], while Davison conducted a study of 2839 patients wherein $1.3 \%$ of the patients were aged $\geq 90$ years [19]. To the best of our knowledge, the current study is the largest cataract surgical study analysing the results of cataract surgery in a very elderly population of people aged 90 years or older. We demonstrate that patients over 90 years of age had a favourable visual outcome following cataract surgery with more than $80 \%$ achieving vision 0.30 LogMAR or better. Poorer outcome results were noted in this population compared to younger patients, alongside a higher rate of PC rupture-vitreous loss. The risk factors reflected those seen in younger patients, and included small pupil, dense/mature cataract and presence of pseudoexfoliation or phacodonesis. The added value of this large population study is that demonstrates that advanced age is a risk factor for poor outcome but not a contraindication for cataract surgery, and surgeons should not be deterred from offering cataract surgery to the very elderly as successful visual rehabilitation occurs.

Surgical techniques and cataract extraction rates per head of population have changed enormously over the past two decades. Technological advances have not been limited to the procedure itself, the current large scale study has been made possible by the use of specialty specific electronic patient record systems, which have allowed the authors to analyse a substantial set of detailed data collected as part of routine clinical care. This has been achieved through a collaborative data sharing exercise with the National Ophthalmology Database Cataract Audit hosted by the Royal College of Ophthalmologists. The included population 
comes from 34 UK cataract surgery centres over a period of 15 years. The size of the reported sample is sufficiently large to allow precise point estimates (percentages and averages).

Many elderly people have ocular and systemic comorbidities which may increase the difficulty of surgery and the complexity of care. Issues may for example frequently arise in regard to comfortable positioning (for patient and surgeon), a higher prevalence of a hard nucleus, a smaller pupil size and a high rate of pseudoexfoliation syndrome. In a UK National Cataract Surgery Survey, one in three participants had a pre-existing ocular condition that could significantly affect cataract surgery outcomes [20]. In that study, AMD was present in $15 \%$ of the participants, glaucoma in $10 \%$ and diabetic retinopathy in $3 \%$. The prevalence of these conditions, as expected, increased with age. In Table 1, we present all the co-morbidities recorded in this surgical population. Among these elderly people AMD was present in $28 \%$ and glaucoma in $11.4 \%$.

In this older group of patients VA outcomes were reduced in comparison with earlier reports on patients unselected for age. Jaycock et al. [21] and Day et al. [11] reported, respectively, that $91 \%$ and $90 \%$ of eyes overall, $95 \%$ and $95 \%$ eyes without co-morbidity, and $82 \%$ and $80 \%$ of eyes with co-morbidity achieved postoperative VA of 0.30 LogMAR $(6 / 12)$ or better in two large UK surgical populations unselected for age. Among the elderly patients reported here this level of VA was achieved by $74 \%$ eyes overall, $85 \%$ eyes without co-morbidity and $64 \%$ eyes with co-morbidity.

In terms of change in VA, Jaycock et al. [21] reported $95 \%$ of eyes as improved or unchanged in patients unselected for age. In the elderly group reported here, $85 \%$ eyes improved, there was no VA change in $10 \%$ and $6 \%$ were recorded as having a worse VA postoperatively. Acknowledging rounding, this was a remarkably similar result to that reported by Jaycock et al. for younger patients. Previous smaller studies on elderly people have reported similar visual changes. Michalska et al. [22], in their study of 122 operations on patients over 90 years of age, reported that postoperative VA improved in $84 \%$ eyes, remained the same in $16 \%$ eyes and deteriorated in $1.6 \%$ eyes, this deterioration being attributed in $62 \%$ of the cases to the underlying advanced AMD. The figure of $84 \%$ for VA improvement in this small study was virtually identical to the $85 \%$ reported here.

There is conflicting evidence whether worse VA outcome after cataract surgery in the most elderly is caused by an independent age factor, or if it can be explained by an increased incidence of age-related co-morbidity [7]. Monestam et al. [23] showed a worse VA outcome in the elderly compared with younger patients, after correcting for the higher percentage of ocular co-morbidity found in elderly people. In the work reported here, risk factors for a clinically important VA Loss identified through multivariate logistic regression analysis indicated that a preoperative diagnosis of AMD or glaucoma significantly increased the risk of VA Loss in this aged population. A good preoperative VA $(\operatorname{LogMAR}=0.30(6 / 12)$ or better) was also a significant risk factor, this perhaps being a ceiling effect as good preoperative VA means that change can only be for the worse. The finding that pre-existing disease is a risk for future VA Loss is not surprising as the natural history of both AMD and glaucoma is progression. Importantly, our study is the first to highlight that age remains a significant risk factor for poorer visual outcome, after adjusting for other factors, including ocular co-morbidities. Awareness of this decline in expected outcome should be useful to clinicians when counselling elderly patients preoperatively to maintain realistic expectations of their surgery, especially in the presence of these co-morbidities.

PCR or vitreous loss or both occurred in $2.7 \%$ eyes. This rate is higher than the multi-centre UK NHS rate for patients unselected for age [11,21], confirming the previously reported higher PCR rate among elderly patients [10]. Specifically, Day et al. reported that the overall rate was $1.95 \%$ across all ages using data from 28 sites between August 2006 and November 2010, a similar time period to that reported here [11]. There have been no further recently reported, adequately powered studies of cataract surgery in people in the age group of interest here.

Our study has some limitations, the most important of which being missing VA data both pre- and postoperatively. Data for VA Loss were only available for $62 \%$ of eyes. This is most likely mainly due to partial uptake of electronic patient record systems that affect the collection of data. Preoperative and operative data are more complete, but postoperative clinical assessments less so. The data analysed were routine high volume surgical service data. Postoperative care pathways vary and some patients are not reviewed back in the surgical provider site. This accounts for a higher level of postoperative missing VA data. Furthermore, postoperative vision testing may take place before patients have received their postoperative spectacle correction and VA testing is mostly undertaken by healthcare assistants, variably done as unaided, corrected or with pinhole. In an attempt to compensate for the missing VA data analyses have been presented based on both the available data (reduced sample size) and imputation of missing data (full sample size). Some reassurance is provided by the fact that the models derived without and with imputation converged and resulted in similar parameters (odds ratios). Other less frequent though important postoperative outcomes such as the rate of endophthalmitis, contrast sensitivity and patient-reported outcomes of surgery, have not been analysed. Also, we did not include data 
on experience of surgeon or on techniques used to handle small pupils. This was not attempted because of absence of data or a lack of confidence in the completeness of such data items.

This is the largest cohort study to report the clinical outcome of cataract surgery in the elderly patients of 90 years of age or older. Medical practice must be evidencebased as a result of tight budgets, waiting lists and the need to target resources effectively. The present study is important, as it is necessary to have reliable data to support the decision of whether to recommend an elderly patient to undergo cataract surgery. Our existing work has told us that being 90 or over confers an additional PCR risk after adjusting for other factors. It is likely that surgery for these elderly patients is being preferentially performed by more experienced surgeons on account of their accumulated risk indicators. These include advanced age but also a higher prevalence of ocular co-morbidities. Overall, we have shown that cataract surgery is safe in the very elderly with improvement in vision for the majority of patients. Our study also showed acceptable rates of complications following cataract surgery compared to the UK national average [21].

In conclusion, the current study indicates that cataract surgery in patients 90 years of age and older has good success rates and can be of benefit for these elderly patients. Provided the factors that influence the procedure positively and negatively are taken into account, surgeons and elderly patients can approach cataract surgery with reasonable confidence of a visually beneficial result.

\section{Summary}

\section{What was known before}

- The risk for complications associated with many surgical procedures is positively correlated with age, but surgical outcomes are negatively correlated with age.

- The number of very elderly patients waiting for cataract surgery is predicted to increase. At the present time, however, only a few reports of cataract surgery in patients older than 90 years have been published.

\section{What this study adds}

- We report an analysis of data from 34 cataract surgery sites from the United Kingdom Royal College of Ophthalmologists' National Ophthalmology Database (NOD).
- To the best of our knowledge, we are the first to report the outcome of cataract surgery in patients older than 90 years in a large cohort study.

Acknowledgements Data were collected on behalf of the Healthcare Quality Improvement Partnership (HQIP) by the Royal College of Ophthalmologists (National Ophthalmology Database Audit provider) as part of the National Clinical Audit and Patient Outcomes Programme. We note with deep regret the death of our friend and colleague Robert Johnston, who died in September 2016. Without his inspirational vision, determination and career long commitment to quality improvement in ophthalmology this work would not have been possible.

\section{Compliance with ethical standards}

Conflict of interest The authors declare that they have no conflict of interest.

Publisher's note: Springer Nature remains neutral with regard to jurisdictional claims in published maps and institutional affiliations.

\section{References}

1. Bernth-Petersen P, Ehlers N. Cataract extraction in the 'nineties'. Acta Ophthalmol (Copenh). 1983;61:392-6.

2. Wong TY. Cataract surgery in patients with cataract and age related macular degeneration: do the benefits outweigh the risks? Br J Ophthalmol. 2000;84:1337-8.

3. Grossmann FF, Zumbrunn T, Frauchiger A, Delport K, Bingisser $\mathrm{R}$, Nickel $\mathrm{CH}$. At risk of undertriage? Testing the performance and accuracy of the emergency severity index in older emergency department patients. Ann Emerg Med. 2012;60:317-25.e3.

4. Nabozny MJ, Kruser JM, Steffens NM, Brasel KJ, Campbell TC, Gaines ME, et al. Constructing high-stakes surgical decisions: it's better to die trying. Ann Surg. 2016;263:64-70.

5. Syam PP, Eleftheriadis H, Casswell AG, Brittain GP, McLeod $\mathrm{BK}$, Liu CS. Clinical outcome following cataract surgery in very elderly patients. Eye (Lond). 2004;18:59-62.

6. Stagg BC, Choi H, Woodward MA, Ehrlich JR. Association of social support network size with receipt of cataract surgery in older adults. JAMA Ophthalmol. 2018;136:423-7.

7. Lundstrom M, Stenevi U, Thorburn W. Cataract surgery in the very elderly. J Cataract Refract Surg. 2000;26:408-14.

8. Norregaard JC, Hindsberger C, Alonso J, Bellan L, BernthPetersen P, Black C, et al. Visual outcomes of cataract surgery in the United States, Canada, Denmark, and Spain. Report From the International Cataract Surgery Outcomes Study. Arch Ophthalmol. 1998;116:1095-100.

9. Sparrow JM, Taylor H, Qureshi K, Smith R, Birnie K, Johnston RL. The Cataract National Dataset electronic multi-centre audit of 55,567 operations: risk indicators for monocular visual acuity outcomes. Eye (Lond). 2012;26:821-6.

10. Narendran N, Jaycock P, Johnston RL, Taylor H, Adams M, Tole $\mathrm{DM}$, et al. The Cataract National Dataset electronic multicentre audit of 55,567 operations: risk stratification for posterior capsule rupture and vitreous loss. Eye (Lond). 2009;23:31-7.

11. Day AC, Donachie PH, Sparrow JM, Johnston RL. The Royal College of Ophthalmologists' National Ophthalmology Database study of cataract surgery: report 1, visual outcomes and complications. Eye (Lond). 2015;29:552-60. 
12. Glynn RJ, Rosner B. Regression methods when the eye is the unit of analysis. Ophthalmic Epidemiol. 2012;19:159-65.

13. Pizzarello LD. The dimensions of the problem of eye disease among the elderly. Ophthalmology . 1987;94:1191-5.

14. Whitmore WG. Eye disease in a geriatric nursing home population. Ophthalmology . 1989;96:393-8.

15. Lim AS, Khoo CY, Ang BC, Tan J, Heng LK. Eye diseases in the elderly in Singapore. Ann Acad Med, Singap. 1987;16:46-53.

16. Gibson JM, Rosenthal AR, Lavery J. A study of the prevalence of eye disease in the elderly in an English community. Trans Ophthalmol Soc U Kingd. 1985;104(Pt 2):196-203.

17. Jay JL, Devlin ML. The increasing frequency of surgery for cataract. Eye (Lond). 1990;4(Pt 1):127-31.

18. Guzek JP, Holm M, Cotter JB, Cameron JA, Rademaker WJ, Wissinger DH, et al. Risk factors for intraoperative complications in 1000 extracapsular cataract cases. Ophthalmology. 1987;94:461-6.
19. Davison JA. Acute intraoperative suprachoroidal hemorrhage in extracapsular cataract surgery. J Cataract Refract Surg. 1986;12:606-22.

20. Gray CS, Crabtree HL, O'Connell JE, Allen ED. Waiting in the dark: cataract surgery in older people. BMJ. 1999;318:1367-8.

21. Jaycock P, Johnston RL, Taylor H, Adams M, Tole DM, Galloway $\mathrm{P}$, et al. The Cataract National Dataset electronic multi-centre audit of 55,567 operations: updating benchmark standards of care in the United Kingdom and internationally. Eye (Lond, Engl). 2009;23:38-49.

22. Michalska-Malecka K, Nowak M, Gosciniewicz P, Karpe J, Slowinska-Lozynska L, Lypaczewska A, et al. Results of cataract surgery in the very elderly population. Clin Interv Aging. 2013;8:1041-6.

23. Monestam E, Wachmeister L. Impact of cataract surgery on the visual ability of the very old. Am J Ophthalmol. 2004;137:145-55. 